\section{A miraculous escape}

A 47 year old housewife walked into hospital one morning complaining of severe pain in her neck and supporting her head with one hand. The previous morning she had tripped and fallen down the stairs but had not lost consciousness. She had no other complaints of pain nor of altered sensation in the limbs. General and neurological examination were unremarkable, but radiographs of the cervical spine (figure) showed a complete fracture-dislocation of the second cervical vertebra on the third cervical vertebra. She had bilateral laminar fractures, and the body of the second cervical vertebra was lying completely anterior to the body of the third cervical vertebra and almost at the same level, with resulting foreshortening of the neck.

Cone calipers were inserted, and skull traction started with the patient nursed on a Stryker frame. Over the next four days weights were gradually increased to $6 \mathrm{~kg}$; further radiographs showed reduction of the dislocation. On the 20th day the patient was taken to the operating theatre still on the frame and her vertebrae fused. Afterwards the patient was nursed on the Stryker frame for five weeks and skull traction was maintained. She was discharged home after 36 days and remains symptom free.

Although the spinal canal is wide at the level of the second cervical vertebra, a displacement of the extent seen in this patient would normally have fatal neurological complications; I have found no published report of a similar case with such a fortunate prognosis.J DOUGLAS BROWN, Orthopaedic Department, Ayr County Hospital, Ayr KA7 3AY.

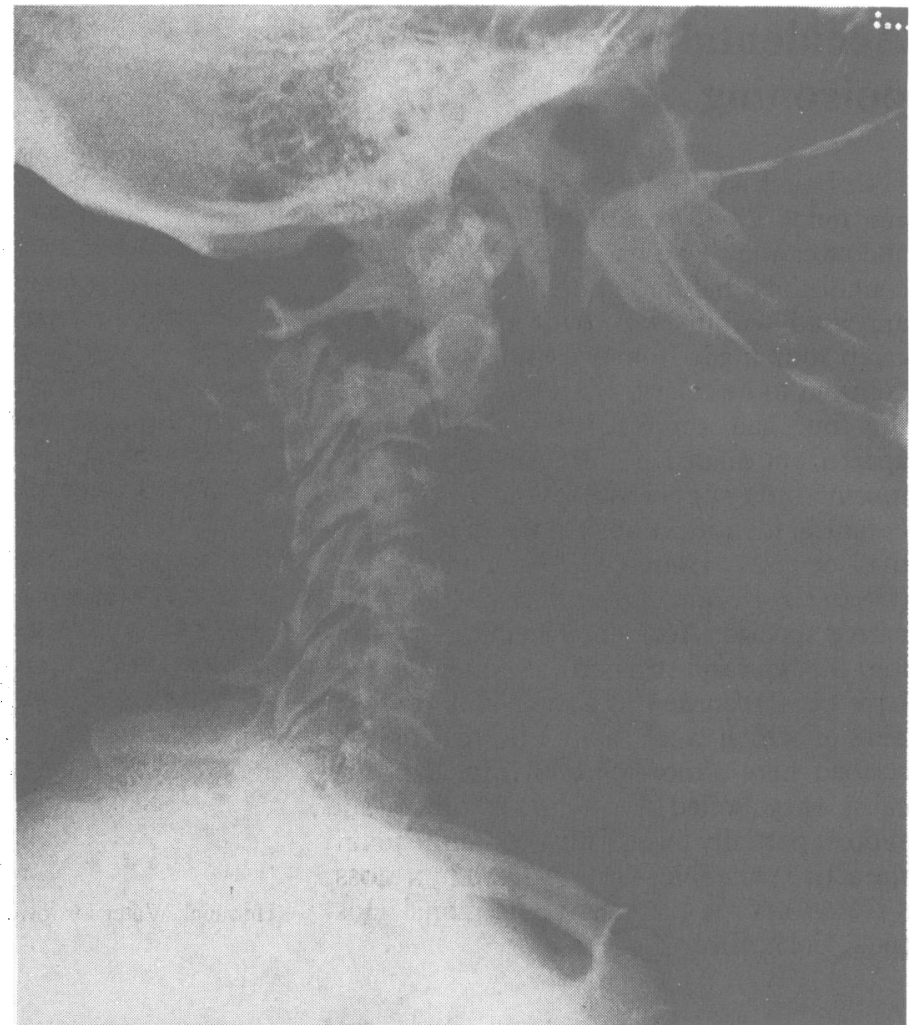

\title{
Cocktail stick injuries: delayed diagnosis of a retained foreign body
}

The dangers of blackthorn injuries are well recognised. ${ }^{1}$ The protruding thorn is removed, but the tip is easily broken off leaving a radiolucent foreign body to cause infection. An equivalent of the blackthorn is the cocktail stick-a hard, sharp wooden spike $8 \mathrm{~cm}$ in length for piercing food.

\section{Case 1}

A 32 year old housewife trod on a cocktail stick while walking barefoot on a carpet. She removed the protruding stick from the sole of her foot and attended the local casualty department for a tetanus toxoid injection. For four months she had recurrent attacks of cellulitis and discharge. Plain $x$ ray films of the foot taken twice showed no foreign body. Her general practitioner and the casualty officer prescribed several courses of different antibiotics. She was referred to the fracture clinic, where examination showed a discharging sinus with a line of nodularity and tenderness on the sole. A sinogram showed a $7 \mathrm{~cm}$ track running subcutaneously. The sole was explored and the remaining $5 \mathrm{~cm}$ of cocktail stick removed. She made a full recovery.

\section{Case 2}

A 12 year old girl had sudden pain in her foot while walking barefoot on a carpet. She removed part of a cocktail stick from between her toes, then attended the local casualty department for a tetanus toxoid booster injection. She reattended two weeks later because of continued pain in the foot and was referred to the orthopaedic registrar who had seen the patient in case 1 three weeks previously. The entry wound, though still visible, was unremarkable, but there was mild diffuse tenderness of the forefoot. At operation $4 \mathrm{~cm}$ of cocktail stick was removed from the web space. She made an uneventful recovery.

\section{Case 3}

A $1^{1 / 2}$ year old girl fell onto a cocktail stick, which entered the hypothenar eminence of her hand. Though her mother thought that she had removed all of the stick, she brought the child to hospital for a tetanus toxoid injection. The wound was dressed, and the child was given a five day course of antibiotics. The wound, however, kept discharging. For the next three months she was given repeat courses of penicillin to control the infection. An abscess developed deep to the entry wound, which was improperly lanced in the casualty department using local anaesthesia. One week later she had cellulitis of the hand and was feverish and unwell. She was admitted to the orthopaedic department and treated with elevation and intravenous penicillin. An allergic rash developed, requiring a change to erythromycin. Her symptoms improved with intravenous treatment, but a tense infective discharging lesion remained in the hypothenar eminence. Finally, under general anaesthesia and using a tourniquet $3 \mathrm{~cm}$ of cocktail stick was removed, the abscess cavity gently curetted, and the wound left open to drain. The hand rapidly healed with no further treatment

\section{Comment}

In each case the cocktail stick was removed by the patient before attending hospital for a tetanus injection. The medical staff failed to establish how long the stick was and whether there was a point at both ends. The patients thought that all of the stick had been removed, but none remembered whether the piece had had a point at both ends. These incidents were considered to be superficial "splinter injuries" and not "penetrating injuries." The wound should be explored if a broken stick is removed; if this is in doubt the wound should be seen again in five to seven days. $x$ Ray films are of little use as wood is radiolucent. Antibiotics will mask the signs of a retained foreign body. If infection develops after a penetration injury this is an indication to explore the wound, not to give repeat prescriptions of antibiotics.

1 Kelly JJ. Blackthorn inflammation. $f$ Bone $y_{t}$ Sirg 1966;48B;474-7.

Department of Orthopaedics, United Medical and Dental Schools of Guy's and St Thomas's Hospitals, London SE1

C RAND, MB, FRCSED, orthopaedic registrar

Correspondence to: Orthopaedic Department, Lewisham Hospital, London SE13. 